\title{
Two-dimensional fluid-filled closed-cell cellular solid as an acoustic metamaterial with negative index
}

\author{
V. Dorodnitsyn* \\ Institute of Mechanical Engineering, École Polytechnique Fédérale de Lausanne, EPFL, CH-1015 Lausanne, Switzerland \\ B. Van Damme ${ }^{\dagger}$ \\ Empa, Swiss Federal Laboratories for Materials Science and Technology, Überlandstrasse 129, CH-8600 Dübendorf, Switzerland
}

(Received 19 January 2016; revised manuscript received 2 March 2016; published 1 April 2016)

\begin{abstract}
A concept for acoustic metamaterials consisting of a cellular medium with fluid-filled cells is fabricated and studied experimentally. In such a system, the fluid and solid structure explicitly interact, and elastic wave propagation is coupled to both phases. Focusing here on shear wave behavior, we confirm previous numerical studies in three steps. We first measure the material deformations pertaining to three qualitatively different shear wave modes in the frequency range below $3.5 \mathrm{kHz}$. We then measure the group velocity and demonstrate that, within a certain frequency interval, the group and phase velocity have opposite signs. This shows that the system acts as a negative-index metamaterial. Finally, we confirm the presence of band gaps due to the locally resonant behavior of the cell walls. The demonstrated concept of a closed, fluid-filled cellular material as an acoustic metamaterial opens a wide space for applications.
\end{abstract}

DOI: 10.1103/PhysRevB.93.134302

\section{INTRODUCTION}

Man-made structures with internal periodicity have received much attention over the past two decades. Deliberately selected mechanical properties are achieved at the macroscale from the geometry of mesoscale cells ordered in a lattice. Possibly the most studied effect is the attenuation of waves in certain frequency ranges, called band gaps $[1,2]$. In phononic crystals, the band gap results from Bragg scattering at wavelengths comparable to the characteristic size of a unit cell [3]. Resonant scattering due to in-lattice resonators can also result in forbidden frequencies, in which case the material is called an acoustic metamaterial [4,5]. Resonance-based dispersion opens the possibility of obtaining even more mechanical properties that cannot be found in nature: a negative refractive index [6-8], acoustic lenses [9,10], acoustic cloaking [11], and wave guiding [12-14].

Cellular materials, artificial or natural, represent a possible class of metamaterials if they have a periodic architecture [15]. In saturated porous media, the two phases of solid and fluid affect the wave propagation, as compared to the solid structure without fluid [16]. The fluid-structure interaction can be tuned by changing mechanical and geometrical properties of both phases. In the present study, fluid flow between the cells is restricted due to the closed-cell configuration. The thin solid structural components play the role of resonators. The horizontal and vertical cell walls influence each other, but they can nevertheless vibrate with different beam modes [17]. In recent studies, we developed numerical and analytical models for wave propagation in two-dimensional (2D) $[17,18]$ and three-dimensional (3D) [19] fluid-filled cellular solids. These analyses demonstrated, in principle, that saturated porous media can act as acoustic metamaterials. They provided

\footnotetext{
*vladimir.dorodnitsyn@epfl.ch

†bart.vandamme@empa.ch
}

guidelines for the design of such systems, e.g., for performance in the audible frequency range.

Here, we experimentally confirm the predictions of the numerical studies, turning concept into reality. We focus on shear waves in order to investigate the presence of band gaps for this wave type. According to the simulations, pressure waves are admitted for all frequencies; therefore the band gaps are called partial [20]. We measure the shear wave-mode deformations, recorded using single-frequency excitation, we use a time of arrival analysis to measure the group velocity of selected wave modes, and finally we identify band gaps and their frequency ranges. All of our findings are qualitatively and quantitatively in agreement with predictions of the prior theoretical analyses.

We first review the finite element (FE) model predictions for the propagation of elastic waves in a $2 \mathrm{D}$ square lattice [Fig. 1(a)]. The presence of fluid in a representative volume element [RVE, shown in the right part of Fig. 1(a)] was implemented by employing an explicit fluid-structure interaction scheme [21, Chap. 12]. Imposing periodic boundary conditions along the high symmetry directions in the irreducible Brillouin zone (iBZ) allows for the computation of the band structure [22]. The cell walls are discretized with two-dimensional plane QM6 elements to avoid shear locking [21, Chap. 6] and to capture thickness deformations of the solid material [18]. The band structure is limited to the path $\Gamma-X$ in the iBZ [horizontal direction of direct space as shown in Fig. 1(a)], and corresponds to the case of water as an entrained fluid, and a wall thickness of $1 \mathrm{~mm}$. The predicted dispersion curves can be assigned to pressure waves (dashed lines) and shear waves (solid lines) [17,23]. A main feature of the latter is the equivoluminal deformation of a unit cell. Typical cell deformations for the first three shear modes are shown in gray in Figs. 1(b), 1(c), and 1(d) for wave vector frequency-combinations corresponding to the experiment described below. It can be seen that the low-frequency shear modes [Figs. 1(b) and 1(c)] require much smaller bending of the vertical walls than of the horizontal walls. The first 


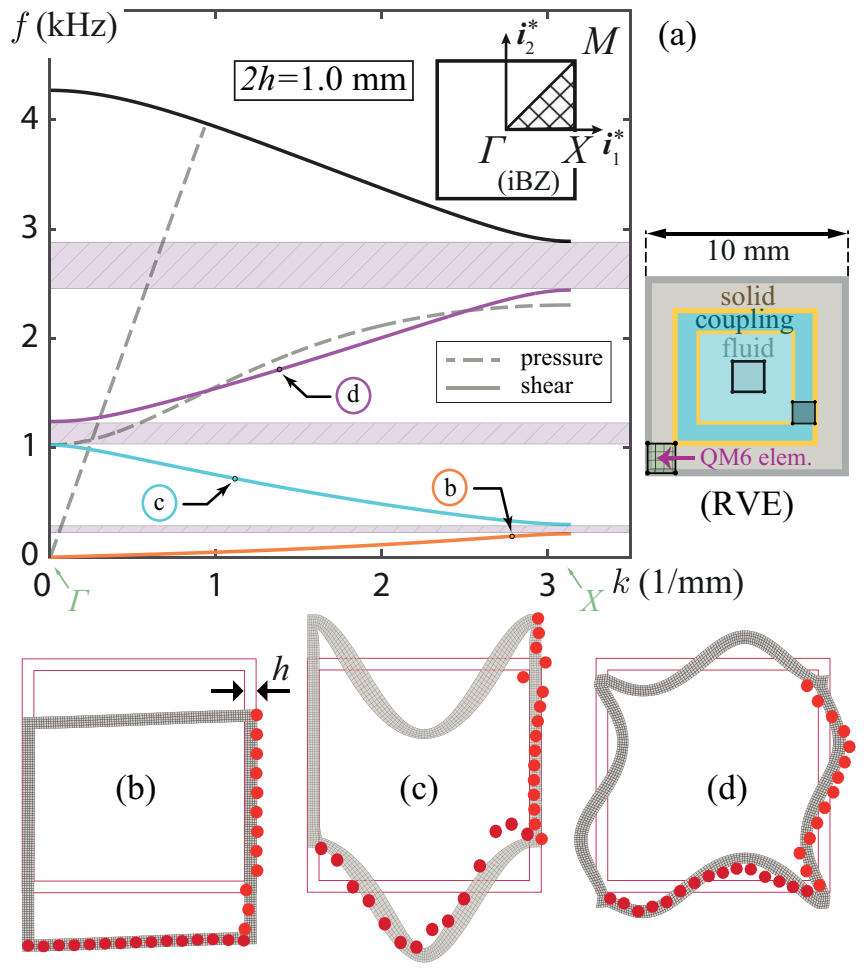

FIG. 1. Band structure (a) for an infinite cellular material with a wall thickness of $1 \mathrm{~mm}$ along the $\Gamma-X$ path in the iBZ, with discretization of an RVE to the right. The band-gap zones are shaded. (b), (c), (d) Deformed configurations of the first three shear wave modes. Numerical wave modes are in gray, while experimental data are superposed with dots (an undeformed configuration is shown with purple frames).

and third modes [Figs. 1(b) and 1(d)] show an antisymmetric deformation of the horizontal walls. For the second shear mode [Fig. 1(c)], the horizontal walls demonstrate a symmetric mode of a beam with rotation-fixed ends. For each consecutive shear wave band, a higher bending mode is excited. The vertical walls can only undergo antisymmetric deformations (with undeformed walls as a limit state) due to the periodic boundary conditions.

The dispersion curves of even shear bands have a negative slope, a property linked to so-called left-handed materials. In these frequency bands, the material has a negative effective mass density and elastic modulus. As a result, the group and phase velocities have opposite signs. Such effective material parameters can be achieved through an interaction of different resonance types [24]. Although a full analytical treatment for cellular materials is beyond the scope of this paper, we expect that the combination of symmetric and antisymmetric cell wall deformations results in this peculiar dynamic behavior. When both cell walls show antisymmetric modes, a right-handed material with positive effective parameters is obtained.

\section{EXPERIMENTAL SETUP}

The design of an experimental cellular solid requires several practical considerations. The structural material properties must be related to the properties of the entrained fluid. To

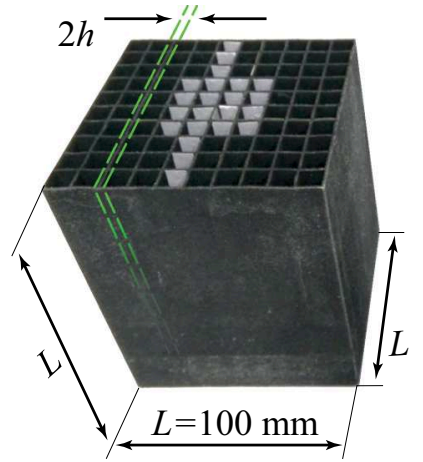

(a)

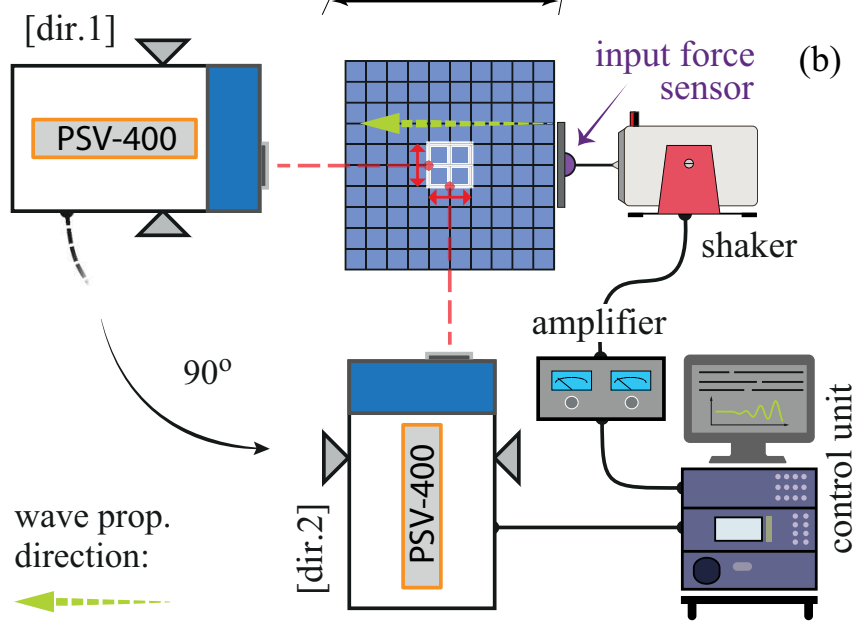

FIG. 2. Experimental setup. (a) The geometry of a sample with wall thickness $2 h$. (b) Scheme of a setup with the rotatable 2D-SLDV scanning head.

guarantee dispersion via resonant scattering, the resonance frequencies of the lattice walls must be well below those of the cavities [18]. To operate at acoustic wavelengths, the cell dimensions must also be chosen appropriately. Here, we use water for the fluid phase (density $\rho_{f}=1000 \mathrm{~kg} / \mathrm{m}^{3}$, bulk modulus $\beta=142 \mathrm{kPa}$ ). We therefore chose DuraForm ${ }^{\circledR}$ Flex as the solid phase. DuraForm ${ }^{\circledR}$ Flex is a microporous thermoplastic elastomer, i.e., a soft rubberlike material, that is suitable for selective laser sintering (SLS), a high-accuracy 3D printing technique. However, the material properties depend on the entrained fluid because some fluid is absorbed into the pores. The elastic modulus and Poisson's ratio of DuraForm ${ }^{\circledR}$ Flex in the water-wet condition were obtained via standard stress-strain tests in accordance with the ASTM D638-14 method [25], with deformations measured via digital image correlation. In the regime of small strains (axial deformation less than 5\%), the material response is nearly linear with Young's modulus and Poisson's ratio $E_{s}=10.38 \mathrm{MPa}$ and $v_{s}=0.28$, respectively. The mass density in soaked conditions was measured to be $\rho_{s}=595 \mathrm{~kg} / \mathrm{m}^{3}$.

Three cellular structures were fabricated having $10 \times 10$ square unit cells in the cross section, with cell dimensions of $1 \times 1 \mathrm{~cm}$ and wall thicknesses $2 h$ of $1,1.5$, and $2 \mathrm{~mm}$, and a height of $10 \mathrm{~cm}$, as shown in Fig. 2(a). This configuration is sufficient to approximate periodic boundary conditions and plane-strain conditions. The cells in the sample are filled with water to within several millimeters of the top of the sample, 
which remains open and unconstrained. The bottom of the sample is fixed to avoid undesired rigid body shifts.

The experimental setup is shown schematically in Fig. 2(b). In order to measure wall deformations, we employed a scanning laser doppler vibrometer (SLDV) (Polytec ${ }^{\circledR}$ PSV-400, operated with the Polytec ${ }^{\circledR}$ PSV-A-010 workstation). The SLDV allows for the reconstruction of the out-ofplane motion of a preselected 2D grid of points. To measure the motion of the walls both perpendicular and parallel to the direction of wave propagation, the SLDV was located in two positions as shown in Fig. 2(b) ([dir.1] and [dir.2], respectively). Scanning was performed on a $2 \times 2$ set of cells in the middle of the sample, ensuring retention of periodic boundary conditions. The scanned area was coated with reflective tape [Fig. 2(a)] to increase the quality of the laser beam reflection. The plane acoustic waves were generated with an LDS V201 shaker. A force sensor was located at the junction of the stinger and percussive plate.

\section{EXPERIMENTAL RESULTS AND DISCUSSIONS}

\section{A. Wall deformation analysis}

For the deformation analysis, we excite the $1 \mathrm{~mm}$ wall sample using a continuous excitation at frequencies corresponding to different expected shear wave modes (200, 700 , and $1700 \mathrm{~Hz}$ ) until the measured velocity reaches a constant level (steady-state regime). To remove noise, the SLDV measurements were averaged 20 times. The resulting velocity signals give an image of the wall deformation in time. Examining a single time frame, we obtain a very good match between experiment and theory, as shown in Figs. 1(b) to 1(d).
Note that, due to the normal excitation, pressure waves are also predicted numerically. We expect the current sample design with slender soft walls to be very compliant for shear waves, compared to the stiffness in the longitudinal direction. Indeed, none of the experimental results shows any trace of pressure waves.

\section{B. Group velocity and negative index behavior}

Using the same cellular structures, we measured the group velocity based on a time of arrival (TOA) analysis. The motion of a wall, away from the boundaries, is measured at a single point of the selected wall using the SLDV in the [dir.1] alignment. For this analysis, the forcing signal consists of a four-cycle sinusoidal pulse modulated with a Hamming window in a frequency range 300-2700 with $50 \mathrm{~Hz}$ steps. The waves are generated by the same shaker connected to a wave form generator (National Instruments PXI 5402) controlled by LabView.

The TOA is measured at the selected wall with respect to the initial force signal recorded by the input force sensor. In order to compare both signals, the recorded wall velocities are smoothened using Savitzky-Golay filtering and then differentiated to obtain the acceleration. Both measured signals, force and acceleration, are wrapped with the envelope. Then, the signals are normalized by the amplitude of the envelope's first peak. Additional peaks might occur in the vibrometer signal due to reflections. Finally, a threshold value is selected at which the time difference is calculated. We chose a midrange value of 0.5. In order to estimate the error in this measurement, several threshold values were chosen as shown in Fig. 3(a) for $2 h=1.5 \mathrm{~mm}$ at $f=1250 \mathrm{~Hz}$. Knowing the TOA and
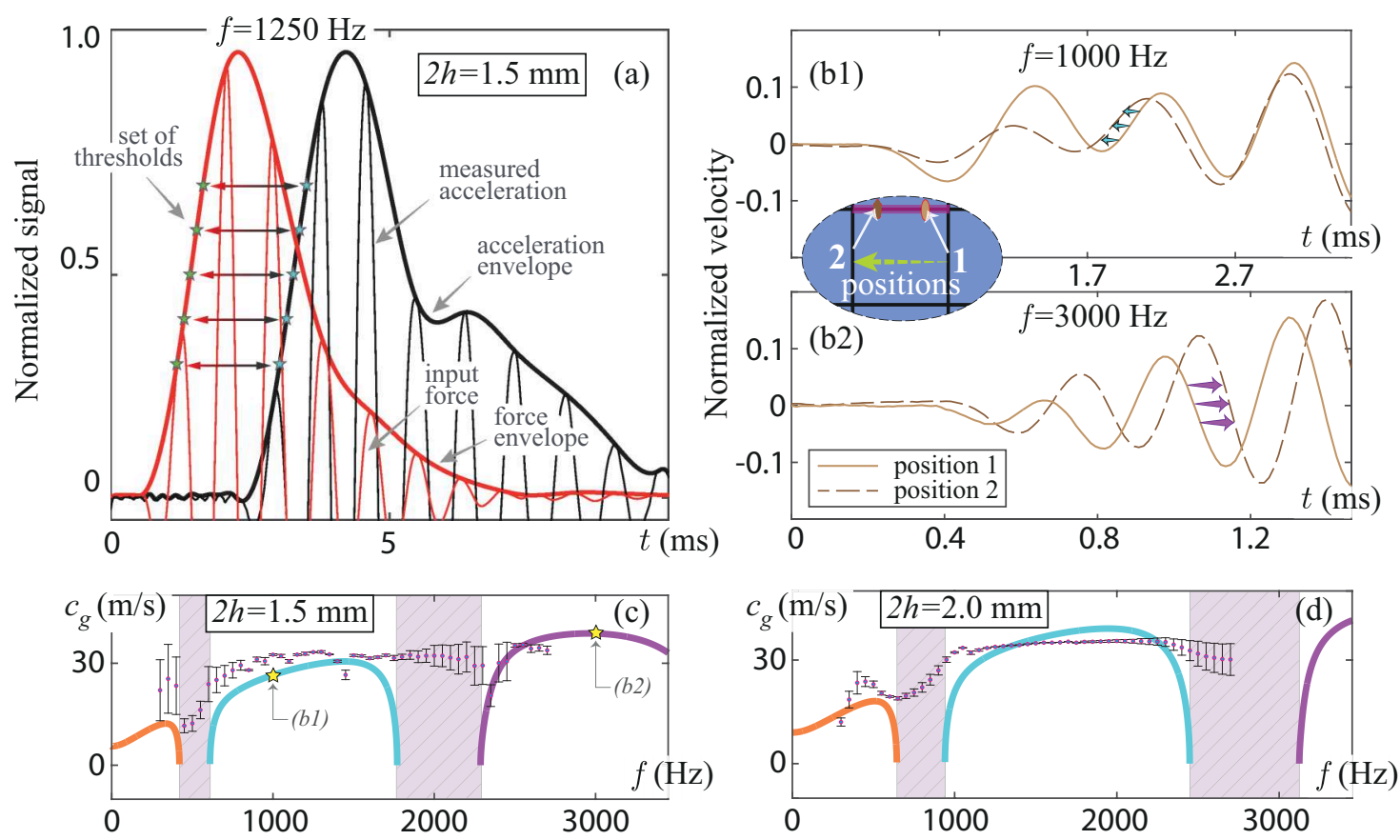

FIG. 3. Group velocity analysis. (a) Thresholds for a TOA measurement for $2 h=1.5 \mathrm{~mm}$ and $f=1250 \mathrm{~Hz}$. The wave propagation direction for $2 h=1.5 \mathrm{~mm}$ : (b1) at $f=1000 \mathrm{~Hz}$ (backward, $c_{g}<0$ ) and (b2) at $f=3000 \mathrm{~Hz}$ (forward, $c_{g}>0$ ). The comparison of experimental and numerical group velocities for (c) $2 h=1.5$ and (d) $2 h=2.0 \mathrm{~mm}$. 
the distance between the input force sensor and the selected cell wall, the group velocity $c_{g}$ can be determined. The set of thresholds defines the error bars as the standard deviation of the mean value at each excitation frequency. The results for $2 h=1.5$ and $2 h=2.0 \mathrm{~mm}$ are shown in Figs. 3(c) and 3(d), respectively. The numerical estimation of the group velocity is gathered from the dispersion curves along $[\Gamma-X]$ using the relation $c_{g}=2 \pi(\partial f / \partial k)$.

Good agreement between experimental and numerical group velocities is achieved for $2 h=1.5$ and $2 h=2.0 \mathrm{~mm}$ [Figs. 3(c) and 3(d)]. The measured velocities show a clear dip for frequencies in the first band gap. In the second band gap, the error bars become considerably larger. This is due to the fact that the resulting pulse contains frequencies from both pass bands around the band gap, which are traveling at different velocities.

One can verify the presence of a left-handed behavior qualitatively based on phase considerations. To do this, we point the laser vibrometer in the [dir.2] direction. Two sensing positions on a single cell wall are shown in the inset of Figs. 3(b1) and 3(b2). Position 1 is closer to the shaker while position 2 is located within the same cell but further from the source. The recorded signals for both positions are shown in Figs. 3(b1) and 3(b2) $(2 h=1.5 \mathrm{~mm}$ at $f=1000$ and $f=3000 \mathrm{~Hz}$ ). At the lower frequency, group and phase velocities are expected to have opposite signs. The phase front measured closest to the source arrives later than the phase front at the more-distant point. Although the wave package travels forward in time, the phase seems to go in the opposite direction, meaning that $c_{p}$ and $c_{g}$ have a different sign. At the higher frequency, positive group and phase velocities are obtained: the direction of motion of the phase is equal to the one of the wave package. The phenomenon of group and phase velocities having opposite different signs is due to a negative refractive index, as has been reported for several other types of acoustic metamaterial configurations $[8,26]$.

\section{Bandgap analysis}

Finally, we confirm experimentally the presence of the band gaps predicted by the FE model. As discussed earlier, these cellular structures are of the local resonant scattering type. Band gaps occur in the vicinity of the resonant frequencies of the beam walls, where destructive interference forbids the propagation of shear waves for any wave number; pressure waves do propagate, however. The numerically predicted band gaps are indicated by the shaded areas in Figs. 3(c) and 3(d). The band gaps here are narrow compared to other metamaterials $[27,28]$. To uncover band gaps experimentally, we study the response of the structure to both a three-period burst and a sweep over the frequency range of interest. The sweep signal is used to verify the band-gap zones whereas the burst measurements clarify the signal deformation due to the dispersion. As an illustrative example, the case $2 h=1.5$ $\mathrm{mm}$ at $f=550 \mathrm{~Hz}$ is shown in Fig. 4(a). The force and wall response used in the TOA experiment are processed via fast Fourier transforms (FFTs) in Matlab to determine the single-sided spectrum. The spectra are plotted on top of each other, and a clear band gap in the vicinity of the selected frequency is observed [Fig. 4(a)]. Since the gap is narrow, the
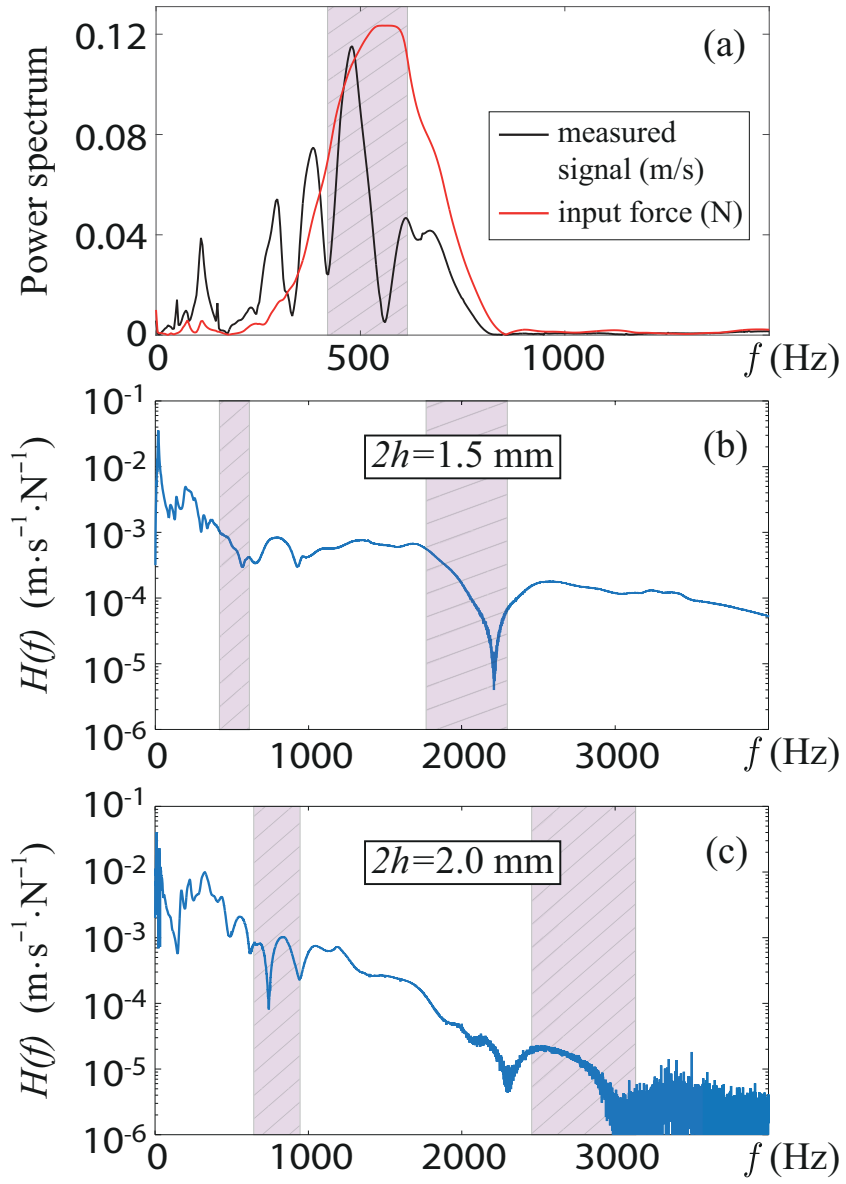

FIG. 4. Band-gap analysis. (a) Spectrum of a single frequency burst for $2 h=1.5 \mathrm{~mm}$ at $f=550 \mathrm{~Hz}$. (b), (c) Transfer function of a sweep signal for $2 h=1.5$ and $2 h=2.0 \mathrm{~mm}$, respectively. The numerical band-gap zones are superposed on top.

remaining frequency content of the pulse can travel through the structure.

The sweep response over an interval $200-4000 \mathrm{~Hz}$ is measured via SLDV on four adjacent walls perpendicular to the wave propagation, and located six cells away from the source, using the SLDV in the [dir.1] orientation. In 20 evenly spaced points, the sweep transfer function $H(f)$ is calculated as the ratio of the measured velocity spectrum and the input force spectrum. The transfer functions for both samples are shown in Figs. 4(b) and 4(c) along with the numerically estimated band gaps. Good agreement between numerical and experimental results is observed. Although the band gaps are narrow and only valid for shear waves, a clear dip in the transfer functions is present. The second band gap is measured at slightly lower frequencies for $2 h=2.0 \mathrm{~mm}$. This can be explained since no damping is applied in the model, although viscoelastic damping becomes more important for thicker cell walls at high frequencies.

\section{CONCLUSIONS}

In summary, we have presented an experimental study of shear wave propagation in an acoustic metamaterial with 
entrained fluid, and confirmed predictions of a previous numerical model. Comparisons were made for the steady-state mode shapes of the shear waves, for the group velocity-in particular regimes of left-handed behavior-and for band gaps in the band structure. For different wall thicknesses, very good agreement with numerical estimates was found for all three of the above features. Therefore, we believe that this work establishes an alternative concept for acoustic metamaterials: a closed-cell cellular solid with entrained fluid.

[1] M. S. Kushwaha, P. Halevi, L. Dobrzynski, and B. DjafariRouhani, Phys. Rev. Lett. 71, 2022 (1993).

[2] J. V. Sánchez-Pérez, D. Caballero, R. Mártinez-Sala, C. Rubio, J. Sánchez-Dehesa, F. Meseguer, J. Llinares, and F. Gálvez, Phys. Rev. Lett. 80, 5325 (1998).

[3] M. Sigalas and E. Economou, Solid State Commun. 86, 141 (1993).

[4] L. Fok, M. Ambati, and X. Zhang, MRS Bull. 33, 931 (2008).

[5] Z. Liu, X. Zhang, Y. Mao, Y. Zhu, Z. Yang, C. Chan, and P. Sheng, Science 289, 1734 (2000).

[6] F. Bongard, H. Lissek, and J. R. Mosig, Phys. Rev. B 82, 094306 (2010).

[7] S. H. Lee, C. M. Park, Y. M. Seo, Z. G. Wang, and C. K. Kim, Phys. Rev. Lett. 104, 054301 (2010).

[8] L. Fok and X. Zhang, Phys. Rev. B 83, 214304 (2011).

[9] S. Zhang, L. Yin, and N. Fang, Phys. Rev. Lett. 102, 194301 (2009).

[10] M. Ambati, N. Fang, C. Sun, and X. Zhang, Phys. Rev. B 75, 195447 (2007).

[11] H. Chen and C. Chan, Appl. Phys. Lett. 91, 183518 (2007).

[12] A. Khelif, A. Choujaa, S. Benchabane, B. Djafari-Rouhani, and V. Laude, Appl. Phys. Lett. 84, 4400 (2004).

[13] Y. Pennec, B. Djafari Rouhani, H. Larabi, A. Akjouj, J. N. Gillet, J. O. Vasseur, and G. Thabet, Phys. Rev. B 80, 144302 (2009).

[14] P. Celli and S. Gonella, Appl. Phys. Lett. 107, 081901 (2015).

[15] X. Wang and T. J. Lu, J. Acoust. Soc. Am. 106, 756 (1999).
Such a metamaterial should find wide application due to its low cost, simplicity, versatility in terms of scalability, and ability to be designed to desired macroscopic behavior using different types of fluids and bulk materials.

\section{ACKNOWLEDGMENTS}

The authors thank S. Kohler and G. Frossard for their kind assistance in tensile measurements, and Prof. W. Curtin for a critical reading of this manuscript.

[16] J. Carcione, in Wave Fields in Real Media: Wave Propagation in Anisotropic, Anelastic, Porous and Electromagnetic Media, Handbook of Geophysical Exploration: Seismic Exploration, Vol. 38 (Elsevier Science, Oxford, 2007).

[17] V. Dorodnitsyn and A. Spadoni, J. Vib. Acoust. 136, 021024 (2014).

[18] V. Dorodnitsyn and A. Spadoni, J. Vib. Acoust. 136, 031005 (2014).

[19] A. Spadoni, R. Höhler, S. Cohen-Addad, and V. Dorodnitsyn, J. Acoust. Soc. Am. 135, 1692 (2014).

[20] Z.-Y. Li, J. Wang, and B.-Y. Gu, Phys. Rev. B 58, 3721 (1998).

[21] R. D. Cook et al., Concepts and Applications of Finite Element Analysis (John Wiley \& Sons, New York, 2001).

[22] C. Kittel, Introduction to Solid State Physics (John Wiley \& Sons, Hoboken, 2005).

[23] A. S. Phani, J. Woodhouse, and N. A. Fleck, J. Acoust. Soc. Am. 119, 1995 (2006).

[24] X. Liu, G. Hu, G. Huang, and C. Sun, Appl. Phys. Lett. 98, 251907 (2011).

[25] ASTM D638-14 (ASTM International, West Conshohocken, PA, 2014).

[26] T. Brunet, A. Merlin, B. Mascaro, K. Zimny, J. Leng, O. Poncelet, C. Aristégui, and O. Mondain-Monval, Nat. Mater. 14, 384 (2015).

[27] S. Krödel, T. Delpero, A. Bergamini, P. Ermanni, and D. M. Kochmann, Adv. Eng. Mater. 16, 357 (2014).

[28] T. Delpero, S. Schoenwald, A. Zemp, and A. Bergamini, J. Sound Vib. 363, 156 (2016). 